\title{
Perceptual and instrumental impacts of robotic laparoscopy on surgical performance
}

\author{
Adélaïde Blavier, ${ }^{1}$ Quentin Gaudissart, ${ }^{2}$ Guy-Bernard Cadière, ${ }^{2}$ Anne-Sophie Nyssen ${ }^{3}$ \\ ${ }^{1}$ National Fund of Scientific Research, Cognitive Ergonomics Laboratory, University of Liège, 5, B32, boulevard du Rectorat, \\ 4000 Liège, Belgium \\ ${ }^{2}$ Department of gastro-intestinal surgery, CHU, St Pierre, Brussels, Belgium \\ ${ }^{3}$ Cognitive Ergonomics Laboratory, University of Liège, Liège, Belgium
}

Received: 16 January 2007/Accepted: 9 February 2007/Online publication: 4 May 2007

New technologies in surgery are in constant and considerable evolution; they transform the surgeon's activity and practice. In laparoscopic surgery, new systems allow the use of two- (2D) or three-dimensional (3D) vision. However, the literature reports contradictory results concerning the benefits of $3 \mathrm{D}$ vision: some studies show that better motor performances are obtained with $3 \mathrm{D}$ vision $[1,8,19,25,26]$ while others fail to reveal any difference in performance between $2 \mathrm{D}$ and $3 \mathrm{D}$ vision $[5,6,12,20]$. In some studies [5], only complex tasks were performed faster and more easily with a 3D view whereas no difference between the use of $2 \mathrm{D}$ and $3 \mathrm{D}$ views appeared when performing the easiest tasks. The divergence in these results is partially due to the fact that first-generation 3D systems, with their lower resolution, were compared with standard 2D systems [10]. Nowadays, new 3D systems allow a natural bidimensional view and thus suppress the bias observed in previous studies.

In this paper, we used a new-generation 3D system, the da Vinci robotic system. This robotic system allows a 3D visualization of the operative field to be gained, restores the degrees of freedom (DOFs) lost in classical laparoscopy, and improves the dexterity of the surgeon's hand and wrist. Only one published study [13] has compared the performance obtained using classical laparoscopic techniques and those using this robotic system. This study revealed advantages of the da Vinci robotic system, particularly when it was used with the $3 \mathrm{D}$ view option. However, only six subjects participated in this study and the chosen tasks were very specific to the robotic system training.

Our objective was therefore to study, with more participants, the impact of the da Vinci robotic system on standard and ecological surgical tasks of increasing

Correspondence to: Adélaïde Blavier complexity (ecological in the sense that our tasks were similar to the gestures made by the surgeon in a real situation, for which we used bench models developed and validated in several studies [7, 22, 23]). To analyse the nature of the benefits brought about by these expensive new technologies precisely, we independently differentiated and studied the influence of the 3D view (afferent component), comparing 2D and 3D view, and the influence of movement freedom restauration (DOFs, efferent component), comparing the classical laparoscopy with the robotic system.

We also studied the impact of the use of the robotic technology on the subject's self-confidence, satisfaction and facility, knowing that these three factors influence both the performance and acceptance of new technology in the operating room $[16,17]$. To avoid any bias from earlier laparoscopic experience in our comparison between classical and robotic laparoscopic techniques, we only selected medical students without any prior experience in open, minimally invasive or robotically assisted surgery.

\section{Materials and methods}

\section{Materials}

The da Vinci system consists of two primary components: the surgeon's viewing and control console and, a moveable cart with three articulated robot arms. The surgeon is seated in front of the console, looking at an enlarged three-dimensional binocular display on the operative field while manipulating handles that are similar to joysticks. Manipulation of the handles transmits electronic signals to the computer, which transfers the same motions to the robotic arms. The computer interface has the capability to control and modify the movements of the instrument tips by downscaling deflections at the handles (by a factor of between 5 and 2). It can also eliminate physiologic tremor, and adjust the grip strength applied to the tools. The computer-generated electrical impulses are transmitted by a 10 meter-long cable and command the three articulated robot arms. 
Table 1. Number of subjects in each condition according to both dimensions

Instrumenta

dimension

\begin{tabular}{llll}
\hline & & $\begin{array}{l}\text { Classical } \\
\text { laparoscopy }\end{array}$ & $\begin{array}{l}\text { Robotic } \\
\text { system }\end{array}$ \\
Perceptive Dimension & 2D & 20 subjects & 20 subjects \\
& 3D & & 20 subjects \\
\hline
\end{tabular}

Disposable laparoscopic articulated instruments are attached to the distal part of two of these arms. The third arm carries an endoscope with dual optical channels, one for each of the surgeon's eyes. As the $3 \mathrm{D}$ visualization can be switched to $2 \mathrm{D}$, we used both the $3 \mathrm{D}$ and $2 \mathrm{D}$ options.

We used a pelvi-trainer for the classical laparoscopic condition (from Ethicon ${ }^{\circledR}$ ). The optical system consists of the laparoscope, the camera, the light source and the video monitor (Storz endoskope ${ }^{\circledR}$ ). The camera was always controlled by the same observer.

\section{Methods}

Sixty medical students ( 26 women and 34 men, mean age $24.9 \pm 2.9$ years) without any prior surgical experience were selected. All subjects underwent a standard acuity examination (with Ergovision and Visuotest from Essilor ${ }^{\circledR}$ ) and only those with either normal or corrected-to-normal vision were included. As shown in Table 1, they were randomly divided into three groups: one performing tasks in classical laparoscopy (pelvi-trainer), another using the robotic system with a 3D view and the third using the robotic system with a $2 \mathrm{D}$ view. The subjects were unaware of the existence of $2 \mathrm{D}$ and $3 \mathrm{D}$ viewing options of the robotic system, and thus unaware of the advantages or difficulties related to their experimental condition.

Our three experimental conditions allowed us to differentiate between two dimensions (as shown in Table 1). We named the first one "perceptive", afferent component, where the da Vinci robotic system, in $2 \mathrm{D}$ and $3 \mathrm{D}$, differed only by the type of vision.

The second dimension was named "instrumental", efferent component. In this dimension, the degrees of freedoms (DOF) were the main difference between the robotic system in $2 \mathrm{D}$ and the classical laparoscopy.

This experimental plan allowed us to study more precisely the influence of this new robotic technology on the surgical performance and, in particular, to answer the following question: is the impact of the da Vinci robotic system explained by the benefits of the 3D vision (in which case we will observe a predominant effect of perceptive dimension and thus a difference between the $2 \mathrm{D}$ and $3 \mathrm{D}$ views) or by the recovery of degrees of freedom (in which case we will observe a predominant effect of the instrumental dimension and therefore a difference between the classical and robotic system, irrespective of the visual dimension)?

\section{Procedure}

The experiment consisted of three phases:

\section{First phase: familiarisation}

Previous studies have shown a strong learning effect after the first use of laparoscopic techniques and in skill learning in general $[2,7,9,14$, 21]. To decrease the learning effect in the subsequent motor tasks and to obtain homogenous groups concerning technical mastery [26], we organised a familiarisation phase. In this phase, subjects repeated a task 10 times (task 0 , see description below) with the technique used in their experimental condition. This phase allowed us to compare the different learning curves according to the type of endoscopic technique.

\section{Second phase: tasks of increasing complexity}

After the familiarisation phase, the subjects performed four tasks of increasing complexity using the technique that they had become familiar with.

\section{Third phase: shift of technique}

In this last phase, subjects performed the most difficult task (task 4) with the technique they had never used: the laparoscopically trained students shifted to the robotic system (10 to the robotic system in 2D and 10 to the robotic system in 3D) and the robotically trained students shifted to the laparoscopic system. Our objective was to study the transfer of a skill acquired with a specific technique to another. Evaluating the performance after a technical switch is highly relevant to understand the risk associated with a change of procedure (e.g., a conversion procedure when the surgeon has to revert to a classical method) to determine an adequate surgical training adapted to the different technologies.

\section{Tasks}

The performance in tasks requiring visual motor control are particularly affected by $2 \mathrm{D}$ vision, whereas the accuracy for verbal judgment or distance estimation is similar with $2 \mathrm{D}$ and $3 \mathrm{D}$ visual systems $[10$, 24]. We therefore selected ecological motor tasks, suitable for novice subjects and compatible with the two techniques. These tasks were selected with the collaboration of an expert surgeon, according to their relevance and validity, which had been demonstrated in previous studies $[7,22,23]$. The five tasks were devised, ranging from basic to more advanced laparoscopic skills. For each task, we calculated a specific performance metric (called the score), which we describe below.

\section{Task 0 (familiarisation task): pick and place}

This task involved grasping and picking up five 5 -mm plastic beads from a starting position, transferring them and dropping them into a receptacle. This task required fine motor skills to grasp the pieces accurately as well as good distance perception to place the pieces into the receptacle accurately. It also required camera moves and allowed to study and develop two-handed video-eye coordination [23]. As only one hand was used in classical laparoscopy, the subjects in this condition performed five trials with the dominant hand and five trials with the non-dominant hand in order to train both hands. At the sixth trial, subjects using the da Vinci robotic system shifted from 2D to 3D or 3D to $2 \mathrm{D}$ and those using the classical laparoscopic technique shifted from the dominant hand to the non-dominant hand (or vice versa).

This task was used in the familiarisation phase and was therefore repeated 10 times.

Performance score: time (in seconds) to put the five pieces into the receptacle.

\section{Task 1: checkerboard}

This task involved arranging 16 rubber letters and numbers into the appropriate squares on a flat surface. It allowed to study spatial relationships on a flat surface and to evaluate accurate fine motor skills [23]. Moreover, this task involved reading letters and numbers, and thus an accurate identification process.

Performance score: number of letters and numbers correctly placed into the squares in four minutes

\section{Task 2: rings route}

This task involved passing a needle through rings. This task required depth perception and wrist articulation skills [23]. It also required particular skill when transferring the needle and therefore good twohanded video-eye-hand coordination. 
Performance score: number of rings the needle went through in four minutes.

\section{Task 3: circular pattern cutting}

This task consisted of cutting a circular pattern. This task involved using the grasper in one hand and applying tension to the material while cutting with the endoscopic scissors in the other hand [7].

Performance score: diameter cut in four minutes, with bonus points if the pattern was cut in less than four minutes.

Penalty score: the cutting accuracy was also evaluated by calculating the percentage area of deviation from the circle outline.

\section{Task 4: suture and knot}

This task involved placing and tying a simple suture using pre-marked points. This task required specific skills when transferring the needle, placing the suture and tying the knot [7]. The suture required manual dexterity to manipulate the instruments and developed two-handed coordination [23]. knot.

Performance score: time (in seconds) to perform both suture and

\section{Questionnaires}

After the familiarisation phase, subjects filled in a questionnaire about their feelings of mastery and familiarity with the technique they used, on a four-point Likert scale.

After the realization of the four tasks of increasing complexity, participants evaluated their performance and answered a questionnaire about their feeling of satisfaction (about their performance), selfconfidence (in their actions and mastery of the system) and difficulty (in the use of the system and the realization of the task) for each task on a four-point Likert scale.

After performing the fourth task with the other technique (technical switch), subjects were asked to compare the two techniques (robotic versus classical laparoscopic system) on a four-point Likert scale and to comment on their general performance. These comments included: speed of task execution, gesture accuracy, gesture quality, image quality, site view, instrument utilization, spatial orientation, comfort, visibility of their actions, difficulty, concentration, quality of feedback of their actions and anticipation of the effect of their actions.

\section{Statistical analysis}

For the familiarisation phase (task 0), the time performance was analysed by a repeated measures analysis of variance. For each task of increasing complexity, an analysis of variance (ANOVA) was used to analyse both performance scores and answers to the questionnaire. We used Newman-Keuls test for the post hoc comparisons. The difference in the answers to the questionnaire in the course of the training was evaluated by a repeated measures analysis of variance to study any change in the subjects' evaluation of satisfaction, self-confidence and difficulty related to the increase of task complexity. We also carried out Pearson correlation analyses between the task-related scores and the scores obtained from questionnaire answers. A Student's $t$-test was used to analyse the answers to the final questionnaire comparing classical laparoscopy with the robotic system. Significance was defined as a $p$ value less than 0.05 .

\section{Results}

\section{Results of the familiarisation phase}

Our results showed that, throughout the familiarization phase, performance was significantly faster with the classical laparoscopic system than with the robotic system (Fig. 1).

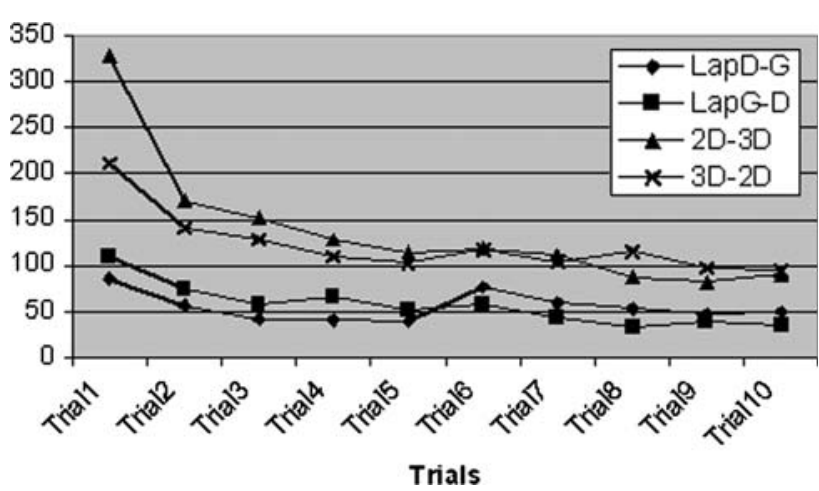

Fig. 1. Learning curves in the four conditions LapD = classsical laparoscopy with dominant hand; LabG = classsical Laparoscopy with non-dominant hand; $2 \mathrm{D}=$ robotic system in $2 \mathrm{D} ; 3 \mathrm{D}=$ robotic system in $3 \mathrm{D}$

We obtained a significant difference in performance between the three conditions in the first trial $(P<0.000)$ : the best performance was observed with subjects using classical laparoscopy followed by those using the robot in 3D and, finally, those using the robot in $2 \mathrm{D}$.

From the second trial on, performance did not statistically differ between the use of the $3 \mathrm{D}$ or $2 \mathrm{D}$ option of the robot; only the difference in performance between the robotic system (2D or 3D) and classical laparoscopy remained the same during the whole familiarisation phase. No significant difference was observed between the use of the two hands in classical laparoscopy.

At the sixth trial, the switch $(2 \mathrm{D} / 3 \mathrm{D}$ for the subjects using the robotic system or dominant/non-dominant hand for subjects in classical laparoscopy) did not provide any significant change in the subject's performance.

The repeated measures analysis of variance showed a significant learning effect during the whole familiarization phase $(P<0.000)$. A significant interaction effect between the conditions in the first five trials emphasized that learning was different according to the technique used $(P<0.000)$. In the last five trials, the learning effect remained $(P<0.000)$ but without any interaction with the type of technique.

Concerning the answers to the questionnaire, there were no differences concerning the feeling of mastery and familiarity, no matter which techniques were used (see Table 2).

\section{Results for the tasks of increasing complexity}

As shown in Table 3, every task was performed significantly better when assisted by the da Vinci robot in the 3D mode than using classical laparoscopy.

In task 1 (checkerboard, $p<0.05$ ), performances were significantly better using the robotic system with a $3 \mathrm{D}$ view than with the robotic system in $2 \mathrm{D}$ and classical laparoscopy.

Performance in task 2 (rings route, $p<0.0000$ ) was significantly different depending on the three experimental conditions: the best performance was observed 
Table 2. Feelings of mastery and familiarity in the three conditions after the familiarisation phase

\begin{tabular}{lllll}
\hline & Classical laparoscopy & Robotic system in 2D & Robotic system in 3D & $P$ value of post hoc analyses \\
\hline Feeling of mastery & $3.05 \pm 0.57$ & $2.85 \pm 0.67$ & $3.13 \pm 0.5$ & No significant difference \\
Feeling of familiarity & $2.41 \pm 0.59$ & $2.37 \pm 0.49$ & $2.69 \pm 0.6$ & No significant difference \\
\hline
\end{tabular}

Table 3. Scores at each task

\begin{tabular}{|c|c|c|c|c|c|c|}
\hline & Classical laparoscopy & ID & Robotic system in $2 \mathrm{D}$ & PD & Robotic system in 3D & $P$ value of post hoc analyses \\
\hline Task 1 & & & & & & \\
\hline $\begin{array}{l}\text { Score } \\
\text { Task } 2\end{array}$ & $7 \pm 2.36$ & & $7.24 \pm 1.95$ & $<$ & $8.94 \pm 2.05$ & $1-3<0.01 ; 2-3<0.05$ \\
\hline $\begin{array}{l}\text { Score } \\
\text { Task } 3\end{array}$ & $2.21 \pm 1.39$ & $<$ & $3.65 \pm 1.62$ & $<$ & $8 \pm 2.15$ & $1-2<0.05 ; 1-3<0.0005 ; 2-3<0.0005$ \\
\hline Score & $69.06 \pm 41.08$ & $>$ & $75.88 \pm 33.7$ & $<$ & $101.31 \pm 34.7$ & 0.05 \\
\hline $\begin{array}{l}\text { Penalty } \\
\text { Task } 4\end{array}$ & $6.89 \pm 3.59$ & $>$ & $4.06 \pm 2.04$ & $>$ & $1.93 \pm 1.28$ & $1-2<0.005 ; 1-3<0.0005 ; 2-3<0.05$ \\
\hline Time & $490.15 \pm 223.04$ & $>$ & $262.21 \pm 114.52$ & & $159.40 \pm 59.13$ & $1-2<0.0005 ; 1-3<0.0005$ \\
\hline
\end{tabular}

$1=$ classical laparoscopy; $2=$ robotic system in $2 \mathrm{D} ; 3=$ robotic system in $3 \mathrm{D}$

$\mathrm{ID}=$ significant influence of instrumental dimension; $\mathrm{PD}=$ significant influence of perceptive dimension

Table 4. Correlations between scores at each task

\begin{tabular}{llll}
\hline & $\begin{array}{l}\text { Task 2: } \\
\text { rings } \\
\text { route }\end{array}$ & $\begin{array}{l}\text { Task 3: } \\
\text { cutting } \\
\text { pattern }\end{array}$ & $\begin{array}{l}\text { Task 4: } \\
\text { suture } \\
\text { and knot }\end{array}$ \\
\hline Task 1: checkerboard & $\begin{array}{l}0.52 \\
\mathrm{p}<0.0000\end{array}$ & 0.2 & -0.19 \\
Task 2: rings route & & 0.36 & $\begin{array}{l}-0.41 \\
p<0.005 \\
-0.24\end{array}$ \\
Task 3: cutting pattern & & $p<005$ & \begin{tabular}{l}
0.24 \\
\hline
\end{tabular} \\
\hline
\end{tabular}

with the robot system in 3D, followed by the performance obtained with the robot in 2D and, finally, the worst performance was obtained using classical laparoscopy.

In task 3 (circular pattern cutting), cut distances were significantly longer with the robotic system in $3 \mathrm{D}$ than with the classical laparoscopy and with the robotic system in 2D $(p<0.005)$.

The cut imprecision (penalty score) was significantly higher with the classical laparoscopic system, followed by the robotic system in $2 \mathrm{D}$, and finally by the robotic system in $3 \mathrm{D}(p<0.00001)$.

In the fourth task, seven subjects were not able to tie the knot in classical laparoscopy conditions. Post hoc analyses only showed differences between the two techniques: the robotic system (in 2D or 3D) led to faster performance than the classical laparoscopy $(p<0.0000)$.

We carried out correlation analyses to study the relationships between the scores for the different tasks. As shown in Table 4, only performance in the second task was significantly correlated with the scores of the other tasks.

Concerning the self-evaluation (Table 5), the ANOVA showed, for each task, that satisfaction (respectively, $p<0.05 ; p<0.05 ; p<0.005 ; p<0.001)$ and selfconfidence (respectively, $\quad p<0.05 ; \quad p<0.000$; $p<0.00005 ; \quad P<0.001)$ were significantly different according to the surgical technique and viewing condition.

The values for the feeling of difficulty only differed in the last task $(p<0.001)$.

When we summed up the subjective scores of all tasks and for each condition (see the ,total line' in Table 5), the subjects felt significantly more satisfied $(p<0.00001)$, self-confident $(p<0.000005)$ and less difficulty $(p<0.005)$ with the robotic system in 3D, followed by the robotic system in $2 \mathrm{D}$, and finally the classical laparoscopic technique.

The repeated measures analysis of variance showed that satisfaction $(p<0.0005), \quad$ self-confidence $(p<0.001)$ and difficulty $(p<0.0000)$ significantly differed between the tasks.

Subjects in classical laparoscopy experienced significantly more difficulties in tasks 2 and 4 than in task 1 (respectively, $p<0.05$ and $p<0.00005$ ). In the 2D robotic system condition, the difficulty estimation differed significantly between tasks 1 and $2(p<0.005)$ while with the robotic system in $3 \mathrm{D}$ no significant differences appeared between the tasks.

Our results in Table 6 showed a significant correlation between the performance scores and their respective subjective evaluations (satisfaction, self-confidence and difficulty), the only exception being the correlation between task 1 and difficulty 1 .

The scores in task 2 showed the strongest correlation with the most self-evaluations and particularly with the total evaluation of satisfaction (0.71), self-confidence (0.61) and difficulty (0.44).

\section{Results of the technical switch}

The ANOVA only showed a significant difference between the classical laparoscopy and the robotic system 
Table 5. Satisfaction, self-confidence and difficulty scores for each task

\begin{tabular}{|c|c|c|c|c|c|c|}
\hline & Classical laparoscopy & ID & Robotic system in 2D & PD & Robotic system in $3 \mathrm{D}$ & $P$ value of post hoc analyses \\
\hline \multicolumn{7}{|l|}{ Task 1} \\
\hline Satisfaction & $2.22 \pm 0.74$ & & $2.31 \pm 0.79$ & $<$ & $2.94 \pm 0.68$ & $1-3<0.05 ; 2-3<0.05$ \\
\hline Self-confidence & $2.55 \pm 0.78$ & & $2.75 \pm 0.58$ & & $3.12 \pm 0.73$ & $1-3<0.05$ \\
\hline Difficulty & $2.38 \pm 0.69$ & & $2.11 \pm 0.93$ & & $2.2 \pm 0.99$ & No significant difference \\
\hline \multicolumn{7}{|l|}{ Task 2} \\
\hline Satisfaction & $1.78 \pm 0.8$ & & $1.69 \pm 0.8$ & $<$ & $2.5 \pm 0.76$ & $1-3<0.05 ; 2-3<0.05$ \\
\hline Self-confidence & $1.72 \pm 0.75$ & & $2.12 \pm 0.8$ & $<$ & $2.8 \pm 0.75$ & $1-3<0.001 ; 2-3<0.05$ \\
\hline Difficulty & $3.22 \pm 0.64$ & & $3.23 \pm 0.84$ & & $2.8 \pm 0.71$ & No significant difference \\
\hline \multicolumn{7}{|l|}{ Task 3} \\
\hline Satisfaction & $2.11 \pm 0.83$ & $<$ & $2.56 \pm 0.89$ & $<$ & $3.18 \pm 0.65$ & $1-2<0.05 ; 1-3<0.005 ; 2-3<0.05$ \\
\hline Self-confidence & $1.94 \pm 0.8$ & & $2.31 \pm 0.7$ & $<$ & $3.19 \pm 0.65$ & $1-3<0.0005 ; 2-3<0.005$ \\
\hline Difficulty & $2.78 \pm 0.55$ & & $2.76 \pm 0.75$ & & $2.53 \pm 0.83$ & No significant difference \\
\hline \multicolumn{7}{|l|}{ Task 4} \\
\hline Satisfaction & $1.78 \pm 0.94$ & $<$ & $2.31 \pm 1.07$ & $<$ & $3.06 \pm 0.68$ & $1-2<0.05 ; 1-3<0.001 ; 2-3<0.05$ \\
\hline Self-confidence & $1.83 \pm 0.79$ & $<$ & $2.75 \pm 1.06$ & & $3.06 \pm 0.85$ & $1-2<0.01 ; 1-3<0.001$ \\
\hline Difficulty & $3.66 \pm 0.59$ & $>$ & $2.82 \pm 1.01$ & & $2.53 \pm 0.88$ & $1-2<0.01 ; 1-3<0.005$ \\
\hline Total Satisfaction & $7.47 \pm 2.7$ & $<$ & $8.87 \pm 1.8$ & $<$ & $11.69 \pm 1.42$ & $1-2<0.05 ; 1-3<0.001 ; 2-3<0.001$ \\
\hline Self-confidence & $8.06 \pm 2.15$ & $<$ & $9.94 \pm 1.84$ & $<$ & $12.06 \pm 1.84$ & $1-2<0.01 ; 1-3<0.0005 ; 2-3<0.005$ \\
\hline Difficulty & $12.09 \pm 1.71$ & & $10.89 \pm 1.82$ & $>$ & $9.50 \pm 2.48$ & $1-3<0.000 ; 2-3<0.05$ \\
\hline
\end{tabular}

$1=$ classical laparoscopy; $2=$ robotic system in $2 \mathrm{D} ; 3=$ robotic system in $3 \mathrm{D}$

ID = significant influence of instrumental dimension; PD = significant influence of perceptive dimension

Table 6. Correlations between scores and feelings of satisfaction, self-confidence and difficulty for each task

\begin{tabular}{|c|c|c|c|c|c|c|c|c|c|c|c|c|c|c|c|}
\hline & satisf1 & satisf2 & satisf3 & satisf4 & sattot & certitl & certit2 & Certit3 & Certit4 & certtot & difficl & diffic2 & diffic3 & diffic4 & difftot \\
\hline Task1 & $.61 * * *$ & $.29 *$ & .22 & $.31 *$ & $.41^{* *}$ & $.47^{* * *}$ & $.31 *$ & $.29 *$ & .18 & $.42^{* *}$ & -.19 & -.20 & .07 & -.11 & -.19 \\
\hline Task2 & $.51 * * *$ & $.68^{* * * *}$ & $.48 * * *$ & $.43 * *$ & $.71 * * *$ & $.37 * *$ & $.55^{* * *}$ & $.49 * * *$ & $.37 * *$ & $.61 * * *$ & -.07 & $-.39^{* *}$ & -.11 & -.26 & $-.44^{* * *}$ \\
\hline Task3 & .12 & .18 & $.47 * * *$ & .16 & $.33^{*}$ & -.05 & .19 & $.40^{* *}$ & .14 & .24 & .17 & -.10 & $-.42 * *$ & -.14 & -.12 \\
\hline Task4 & -.15 & -.06 & $-.35^{*}$ & $-.57 * * *$ & $-.45^{* *}$ & -.01 & -.06 & -.23 & $-.54 * * *$ & $-.35^{*}$ & .16 & -.01 & .05 & $.51 * * *$ & $.35^{*}$ \\
\hline
\end{tabular}

${ }^{*} p<0.05 ; * p<0.005 ; * * *<0.000$

sattot, certtot and difftot $=$ sum of all subjective scores of respectively, satisfaction, self-confidence and difficulty

Table 7. Time (in seconds) to execute the suture and the knot after the technical switch

\begin{tabular}{lllll}
\hline & Classical laparoscopy & Robotic system in 2D & Robotic system in 3D & $P$ value of post hoc analyses \\
\hline Task 4 Time & $519.57 \pm 65.94$ & $326.17 \pm 92.68$ & $206.73 \pm 53.84$ & $1-3<0.05$ \\
\hline
\end{tabular}

$1=$ classical laparoscopy; $2=$ robotic system in $2 \mathrm{D} ; 3=$ robotic system in $3 \mathrm{D}$

in $3 \mathrm{D}$ in performing task 4 a second time after the technical switch $(p<0.01$, see Table 7).

The final questionnaire comparing the two techniques showed a significant difference for all items. The only exception was for concentration and the feedback quality, but this might be due to the fact that these two questions were too abstract or might not have been understood by the participants (see Table 8).

\section{Discussion}

\section{Familiarisation phase}

The objective of this phase was to train the subjects to use a specific surgical technique (manipulating instruments, moving the camera, grasping objects, aiming a recipient) to prevent a strong familiarisation effect in subsequent motor tasks. Indeed, we observed a very fast familiarization of the different techniques: the improvement of the performance between the first and second trial was very strong (by $50 \%$ and $30 \%$ with the robotic system in $2 \mathrm{D}$ and $3 \mathrm{D}$, respectively), confirming the existence of a period of rapid initial learning as shown in other studies in surgery and cognitive psychology $[2,5,7,9,14,21]$. However, although all the learning curves reached a plateau at the end of the 10 trials, they followed a different pattern for each technique: as in the Prasad et al. study [21], our results demonstrated an early phase of greater learning with the robotic system (in 2D and 3D), while the learning curve was extremely reduced, nearly nonexistent, in classical laparoscopy, in contrast to other studies that showed strong learning curves in classical laparoscopy [13].

Moreover, in all trials we obtained better performance with the classical laparoscopy than with the ro- 
Table 8. Answers to questionnaire comparing the two techniques (classical and robotic laparoscopy)

\begin{tabular}{lcrl}
\hline & $\begin{array}{l}\text { Classical } \\
\text { laparoscopy }\end{array}$ & \multicolumn{1}{l}{$\begin{array}{l}\text { Robotic } \\
\text { system }\end{array}$} & $t$ and $p$ values \\
\hline General performance & $1.8 \pm 0.8$ & $3.5 \pm 0.63$ & $6.6,<0.00000$ \\
Speed of performance & $1.94 \pm 0.82$ & $3.25 \pm 0.77$ & $4.69,<0.0001$ \\
Gesture accuracy & $1.88 \pm 0.86$ & $3.5 \pm 0.82$ & $5.54,<0.00001$ \\
Image quality & $2.12 \pm 0.69$ & $3.19 \pm 1.05$ & $3.48,<0.005$ \\
Site view & $2.12 \pm 0.69$ & $3.38 \pm 0.96$ & $4.33,<0.0005$ \\
Instrument utilization & $1.71 \pm 0.77$ & $3.69 \pm 0.48$ & $8.79,<0.00000$ \\
Spatial orientation & $2.12 \pm 0.93$ & $3.38 \pm 0.72$ & $4.33,<0.0005$ \\
Comfort & $1.94 \pm 0.85$ & $3.63 \pm 0.72$ & $6.05,<0.000005$ \\
Concentration & $2.24 \pm 0.66$ & $2.44 \pm 1.15$ & Not significant \\
Feedback quality & $2.47 \pm 0.79$ & $2.75 \pm 0.93$ & Not significant \\
Action visibility & $2.12 \pm 0.69$ & $3.44 \pm 0.81$ & $5.04,<0.00005$ \\
Anticipation & $2.18 \pm 0.73$ & $2.88 \pm 0.81$ & $2.37,<0.05$ \\
Complexity & $3 \pm 1.06$ & $1.75 \pm 0.86$ & $3.71,<0.001$ \\
Gesture quality & $2.24 \pm 0.83$ & $3.25 \pm 0.45$ & $4.32,<0.0005$ \\
\hline
\end{tabular}

botic system (in 2D and 3D). Although we observed a significant difference in the first trial between 3D and 2D vision with the robotic system in this relatively easy task, this difference disappeared after the first trial. It seems that, although the 2D vision affected performance at first, subjects rapidly and accurately compensated for the lack of binocular depth perception, relying on only monocular cues (namely light and shade, relative size of objects, object interposition, texture gradient, aerial perspective and, very important, motion parallax) to perform as fast as subjects in the $3 \mathrm{D}$ robotic system.

Two aspects of the task could partially explain the results we observed in classical laparoscopy (best performance, floor effect and absence of learning curves).

First, the task was very easy, and perhaps easier than the tasks used in other studies. This task did not require any specific fine movements, and the manipulation was very basic without any need to grasp pieces in a specific way. This argument could partially explain the absence of learning curves in classical laparoscopy but it cannot account for the better performance observed in classical laparoscopy, as the robotic system was also not advantaged by the easiness of the task.

The second aspect is that the task required frequent camera moves to explore the whole site and grasp all the pieces, whereas the robotic system seems to be particularly adapted to microsurgery where fine suturing and knot tying are required (for example, in our most complex task). The need to move the camera frequently for relatively long distances actually constituted a second task in itself that had to be learned and performed by subjects. This may account for the fact that performance with the robot never caught up with the performance observed in classical laparoscopy. In classical laparoscopy, the movement of the camera does not require a long learning period and can occur simultaneously with the instrument's movement. The robotic system, however, requires a change of mode (pushing a foot pedal and manipulating the same handles as those used for instrument movement) and this has to be performed in succession with instrument movement. Prasad et al. [21] also obtained the same results in their study comparing the learning curves obtained with classical laparoscopy and the Zeus robotic system (2D view). In their study they pointed out that the nature of the task could be a factor contributing to these findings. In our study, the second task of moving the camera influenced and thwarted the advantages of the robotic system, showing the limitations of this technology. This finding is in accordance with clinical and experimental observations concerning the specific advantages brought about by the robotic system in microsurgery or in small operating fields $[3,11,18]$.

Finally, although this familiarisation with a very simple task cannot be considered as a strong expertise acquisition, we noted that this phase allowed our subjects to be confident when performing the subsequent tasks.

\section{Tasks of increasing complexity}

Our results showed that, in all tasks, the robotic system in $3 \mathrm{D}$ led to better performance than classical laparoscopy. Moreover, the difference between the 3D robotic system and classical laparoscopy tended to increase with the difficulty of the tasks. Indeed, the difference in the first, simplest task was smaller and less significant than that observed in the subsequent and more-complex tasks. We also noted a significant difference between the robotic system in 2D and classical laparoscopy in all tasks except for the first, easiest task. All these results are in accordance with the Hubens et al.'s study [13].

If we analyse the impact of the robotic technology in terms of perceptive and instrumental benefits (Table 1), we observe that the influence of the two dimensions differs according to the nature and complexity of the task. The perceptive dimension played a significant role and could explain the performance in the first three tasks. It was also the only determinant factor for performance in the first and easiest task. These findings confirm the important impact of binocular depth perception on surgical performance $[1,8,19,25,26]$.

The influence of the instrumental dimension was significant in the last three tasks - tasks involving morecomplex movements than just grasping. In the fourth task, manual demands overlapped with the perceptive advantage and only the instrumental dimension differentiated between the conditions. Indeed, in classical laparoscopy, the instrument length and rigidity seemed to be the most difficult obstacle to overcome to introduce the needle and particularly to cross the instruments to tie the knot. In this difficult task, only the additional DOF (instrumental dimension) accounted for the difference between the laparoscopic and robotic performance and this difference in the instruments far outweighed the minor difference between $2 \mathrm{D}$ and $3 \mathrm{D}$ vision with the robotic system. The absence of any significant difference between the $2 \mathrm{D}$ and $3 \mathrm{D}$ viewing conditions in the robotic system in this task could also be explained by the fact that both hands were in movement, providing strong motion parallax, which is a particularly efficient monocular cue for depth perception [27].

The two tasks of intermediate complexity (tasks 2 and 3) seemed to involve both perceptive and instrumental dimensions. 
The data from the questionnaires showed the same tendency: subjects generally felt less confident, less satisfied and more difficulty with classical laparoscopy than with the robotic system in $2 \mathrm{D}$, followed by the robotic system in 3D. Self-confidence, satisfaction and facility are determining factors in the acceptance of new technology into the operating theatre [16]. Self-confidence is an important aspect of optimal performance and may lead to increased self-efficacy [17]. Indeed, although overconfidence could be considered a pervasive cognitive bias and thus a negative component, cognitive anxiety is characterized by worry, negative expectations and concentration disruption, and thus could strongly disturb activity [15]. Moreover, self-confidence has a more significant impact upon performance on the surgical clerkship than in other areas of medicine $[4,17]$. In our study, one may assume that our subjects had expectations about the robotic system and anticipated that it would be easier. However, the difference observed between the robotic system in 2D and 3D confirms that self-confidence was influenced by depth perception and was not determined by expectations about the use of the new technology. In the same way, higher satisfaction with the robotic system could be explained by the effect of novelty. However, the difference between the 3D and $2 \mathrm{D}$ views of the robotic system indicated that subjects relied more on their actual performance than on any novelty effect produced by the robotic system.

Moreover, our results showed that satisfaction, selfconfidence and difficulty evolved differently during the tasks and emphasized perceptive and instrumental dimensions. Under classical laparoscopy conditions, the task considered most difficult was task 4 whereas with the robotic system, the most difficult was task 2. This finding (albeit not statistically significant) about the subjective evaluation of difficulty confirms the role of instrumental dimension in task 4 , as emphasized by the performance scores.

Finally, our results also showed that the performance in task 2 was the only one to be significantly correlated to the other tasks: indeed, the rings route task includes a lot of useful and usual fine movements required in minimal invasive surgery and notably reproduced some components of the complexity of the suture gesture (except the knot). Moreover, scores on this task were highly correlated with the subjective evaluation of satisfaction, self-confidence and difficulty. Therefore, this task seems to be a very efficient and accurate way to evaluate minimal invasive systems or to improve and train surgical performance.

\section{Technique switch}

After the technique switch, our results showed better performance with the robotic system in $3 \mathrm{D}$ than with the classical laparoscopy, these two conditions showing no significant difference with the robotic system in 2D. This switch occurred without any learning of the technique. Moreover, the task (suture and knot) was the most difficult one. This result emphasized the role of the two dimensions described in Table 1: both instrumental and perceptive dimensions seemed to be necessarily present and the presence of only one of them was not sufficient to provide any significant difference. In this case, it is thus the combination of the advantages of the binocular vision with the restoration of degrees of freedom (DOF) that led to better performance. The results after this technique switch are ecologically relevant, especially as far as two phenomena linked to surgery are concerned: the problem of conversion procedure and the problem of surgeon's training and formation. Indeed, the extremely bad performance by robotically trained subjects when they had to performed classical laparoscopy after the technique switch emphasizes the risk associated with a conversion procedure performed by a surgeon who has mainly trained with the robotic system. Although there is less risk for conversion with the robotic system (because the hand motions are exactly like those of open surgery), the risks are high for classical laparoscopy due to the fulcrum effect, the 2D view, and the reduced DOF, as confirmed by our data with novice subjects.

\section{General conclusion}

To conclude, our study showed that the robotic system obviously has some advantages: binocular vision in all tasks and movement freedom of the instruments, particularly in fine motor tasks. These advantages were particularly emphasized in small fields because camera movements can be a significant drawback of the robotic system. Moreover, we showed that the lack of depth perception can be compensated by the camera or movements of the hands. On a subjective level, the robotic system provided satisfaction, self-confidence and facility for novice subjects, particularly with $3 \mathrm{D}$ vision. However, the poor performance after the technique switch emphasizes the necessity for training with classical laparoscopic techniques. These contrasting findings emphasize the importance of studying the whole activity and not limiting research to only some aspects of the task.

We showed that the influence of both perceptive and instrumental benefits depended on the complexity and demands of the task. This suggests that the underlying cognitive and motor processes involved in the different tasks are somewhat different. Further studies are necessary to understand better the implication of these different cognitive mechanisms, notably with expert surgeons, to evaluate if visuomotor processes change with expertise. Experienced surgeons are used to operating with a 2D image in classical laparoscopy and have therefore developed compensatory mechanisms using monocular visual cues, which require a lot of practice and a new organisation of the visuomotor system [24]. A fourth experimental condition could also be introduced to complete the study of the involvement of the two dimensions described in the Table 1: classical laparoscopy with direct 3D view (and thus without a camera). In this study, we did not use this condition because of its lack of clinical and ecological relevance. However, integrating this condition into another study would be theoretically relevant. It is important to understand the 
nature of the cognitive and motor processes involved in the execution and control of laparoscopic gestures. Furthermore, this issue could be relevant for the development of both surgical procedures and training, considering safety as well as technological evolution in surgery.

Acknowledgments. The authors would like to thank the medical students who participated in the study and David Douglas and Olivier Germay for their expert technical assistance. This research was supported by a grant from the National Fund of Scientific Research of Belgium. Equipments were provided by Intuitive Surgical, Ethicon, Storz Endoscope and Essilor.

\section{References}

1. Birkett DH, Josephs LG, Este-McDonald J (1994) A new 3-D laparoscope in gastrointestinal surgery. Surg Endosc 8: 1448-1451

2. Brashers-Krug T, Shadmehr R, Bizzi E (1996) Consolidation in human motor memory. Nature 382: 252-255

3. Cadière GB, Himpens J, Vertruyen M, Bruyns J, Germay O, Izizaw R (2001) Nyssen fundoplication. Surg Endosc 15: 918-923

4. Carter MB, Wesley G, Larson GM (2005) Didactic lecture versus instructional standardized patient interaction in the surgical clerkship. Am J Surg 189: 243-248

5. Chan ACW, Chung SCS, Yim APC, Lau JYW, Ng EKW, Li AKC (1997) Comparison of two-dimensional vs three-dimensional camera systems in laparoscopic surgery. Surg Endosc 11: 438-440

6. Crosthwaite G, Chung T, Dunkley P, Shimi S, Cuschieri A (1995) Comparison of direct vision and electronic two- and threedimensional display systems on surgical task efficiency in endoscopic surgery. Brit J Surg 82: 849-851

7. Derossis AM, Fried GM, Abrahamowicz M, Sigman HH, Barkun JS, Meakins JL (1998) Development of a model for training and evaluation of laparoscopic skills. Am J Surg 175: 482-487

8. Dion YM, Gaillard F (1997) Visual integration data and basic motor skills under laparoscopy: Influence of 2-D and 3-D videocamera system. Surg Endosc 11: 995-1000

9. Doyon J, Penhume V, Ungerleider LG (2003) Distinct contributions of the cortico-striatal and cortico-cerebellar systems to motor skill learning. Neuropsychologia. 41: 252-262

10. Falk V, Mintz D, Grünenfelder J, Fann JL, Burdon TA (2001) Influence of three-dimensional vision on surgical telemanipulator performance. Surg Endosc 15: 1282-1288

11. Garcia-Ruiz A, Gagner M, Miller JH, Steiner CP, Hahn JF (1998) Manual vs robotically assisted laparoscopic surgery in the performance of basic manipulation and suturing tasks. Arch Surg 133: $957-961$
12. Hanna GB, Shimi SM, Cuschieri A (1998) Randomised study on influence of two-dimensional versus three-dimensional imaging on performance of laparoscopic cholecystectomy. Lancet 351: 248 251

13. Hubens G, Coveliers H, Balliu L, Ruppert M, Vaneerdeweg W (2003) A performance study comparing manual and robotically assisted laparoscopic surgery using the da Vinci system. Surg Endosc 17: 1595-1599

14. Huber JW, Taffinder N, Russell RCG, Darzi A (2003) The effects of different viewing conditions on performance in simulated minimal access surgery. Ergonomics 46: 999-1016

15. Jones G (1990) A cognitive perspective on the processes underlying the relationship between stress and performance in sport. In: Jones G, Hardy L (eds). Stress and performance in sport Wiley, 1990, Chichester, UK, pp 17-42

16. Jones G, Cale A (1997) Goal difficulty, anxiety and performance Ergonomics 40: 319-333

17. Marshall RL, Smith JS, Gorman PJ, Krummel TM, Haluck RS, Conney RN (2001) Use of a human patient simulator in the development of resident trauma management skills. J Trauma 51: $17-21$

18. Nio D, Bemelman WA, den Boer KT, Dunker MS, Gouma DJ, van Gulik TM (2001) Efficiency of manual vs robotic (Zeus) assisted laparoscopic surgery in the performance of standardized tasks. Surg Endosc 16: 412-415

19. Peitgen K, Walz MV, Holtmann G, Eigler FW (1996) A prospective randomized experimental evaluation of three-dimensional imaging in laparoscopy. Gastrointest Endosc 44: 262-267

20. Pietrabissa A, Scarcello E, Mosca F (1994) Three-dimensional versus two-dimensional video system for the trained endoscopic surgeon and the beginner. End Surg 2: 315-317

21. Prasad SM, Maniar HS, Soper NJ, Damanio RJ, Klingensmith ME (2002) The effect of robotic assistance on learning curves for basic laparoscopic skills. Am J Surg 183: 702-707

22. Reznick R, Regehr G, MacRae H, Martin J, McCulloch W (1997) Testing technical skill via an innovative "bench station" examination. Am J Surg 173: 226-230

23. Scott DJ, Bergen PC, Rege RV, Laycock R, Tesfay ST, Valentine RJ, Euhus DM, Jeyarajah DR, Thompson WM, Jones DB (2000) Laparoscopic training on bench models: Better and more cost effective than operating room experience? J Am Coll Surg 191: 272-283

24. Servos P (2000) Distance evaluation in the visual and visuomotor systems. Exp Brain Res 130: 35-47

25. Taffinder N, Smith SGT, Huber J, Russell RCG, Darzi A (1999) The effect of a second-generation 3D endoscope on the laparoscopic precision of novices and experienced surgeons. Surg Endosc 13: $1087-1092$

26. Van Bergen P, Kunert W, Bessell J, Buess GF (1998) Comparative study of two-dimensional and three-dimensional vision systems for minimally invasive surgery. Surg Endosc 12: 948-954

27. Voorhorst FA, Overbeeke KJ, Smets GJF (1996) Using movement parallax for 3D laparoscopy. Med Prog Technol 21: 211-218 